\title{
LUCHANDO POR SUS DERECHOS EN TIEMPOS DE COVID-19. RESISTENCIAS Y RECLAMACIONES DE REGULARIZACIÓN DE LOS MIGRANTES SINPAPELES EN ESPAÑA
}

\author{
Fighting for Their Rights in Times of Covid-19. \\ Resistance and Claims for Regularization of Sinpapeles Migrants in Spain
}

Ana López-Sala*

\begin{abstract}
Resumen. La Covid-19 ha provocado una profunda crisis social en España que ha infligido una importante herida en las condiciones de vida de la población, pero especialmente en los sectores más vulnerables, entre los que destacan las comunidades inmigrantes. Durante esta crisis sanitaria, la toma de conciencia de los inmigrantes sinpapeles como sujetos esenciales que realizan una aportación imprescindible, pero que son objeto de no-derechos ha cristalizado en una relevante lucha política en torno al movimiento \#RegularizaciónYa. El objetivo de este artículo es analizar el surgimiento y desarrollo de esta movilización social que se enmarca en los denominados estudios críticos de ciudadanía.
\end{abstract}

Palabras clave: Covid 19; Sinpapeles; regularización; activismos migrantes; ciudadanía; España.

\begin{abstract}
Covid-19 has provoked a deep social crisis in Spain that has inflicted a major wound in the living conditions of the population, but especially in the most vulnerable sectors, among which the immigrant communities stand out. During this health crisis, the awareness of immigrants without papers as essential subjects who make an indispensable contribution, but who are the object of non-rights, has crystallized into a relevant political struggle around the movement \#RegularizacionYa. The aim of this article is to analyze the emergence and development of this social mobilization that is framed in the critical citizenship studies and more specifically in the literature on acts of citizenship.
\end{abstract}

Keywords: Covid 19; Sinpapeles; legalization campaign; migrant activisms; citizenship; Spain.

Instituto de Economía, Geografía y Demografía (IEGD), Consejo Superior de Investigaciones Científicas (CSIC). Madrid, España. E-mail: ana.Isala@cchs.csic.es. Orcid: https://orcid.org/00000003-2756-7042. 


\section{Introducción ${ }^{1}$. De invisibles a esenciales}

La crisis sanitaria ocasionada por la COVID 19 ha tenido un enorme impacto en las dinámicas sociales, económicas y políticas en España. Estos efectos han estado en gran parte moldeados por el impacto de la pandemia en la regulación de la movilidad, tanto interna como internacional, que ha conformado un escenario absolutamente imprevisto y completamente inédito en la historia reciente de los estudios migratorios. Si durante décadas la comprensión de los determinantes que activan y mantienen la movilidad han dado forma e impulso a los estudios migratorios en las ciencias sociales, esta "crisis de inmovilidad" (Newland, 2020; Papademetriou, Hooper, 2020) no sólo ha afectado seriamente a nuestras vidas, sino que se ha transformado en un poderoso catalizador para repensar y reformular muchas asunciones, quizás excesivamente asentadas, en el estudio de la movilidad y su gestión.

La crisis sanitaria produjo el cierre de las fronteras de la mayor parte de los países europeos, afectando significativamente a uno de los pilares en los que se basa su estructura económica y productiva actual: la movilidad de trabajadores desde el exterior. El efecto más inmediato en el caso español, también observado en el caso de otros países de Europa, fue una reducción drástica de la inmigración estacional, fundamentalmente la dedicada a la agricultura, que ocasionó un destacado debate político, académico y social sobre un secreto a voces, pero ampliamente invisibilizado durante décadas: la amplia dependencia en el sector agrícola de la mano de obra extranjera y su papel fundamental en la sostenibilidad de este sector económico. En plena crisis sanitaria lo apreciado en el sector agrícola se extendió a otros sectores y a otros trabajadores, ahora etiquetados como esenciales (Anderson et al., 2020): los que se emplean, por ejemplo, en tareas de cuidado, servicios de limpieza y mantenimiento o en las cadenas de distribución de mercancías. Estos sectores, donde la presencia de personas inmigrantes es muy destacada, constituyen, sin embargo, los segmentos más precarizados de la economía española, caracterizados no sólo por las condiciones más duras y los peores salarios, sino por una alta incidencia del empleo informal y la vulneración de los derechos laborales más básicos. Son además sectores en donde a la informalidad económica se une la informalidad legal, ya que en ellos se emplean un buen número de personas migrantes en situación irregular (Lastra, Cachón, 2013).

Es precisamente en este contexto en el que surge, en abril de 2020, una amplia acción política y de resistencia de los inmigrantes sinpapeles en defensa de sus derechos ciudadanos y laborales que se articula a través del movimiento \#RegularizaciónYA. Se trata de una movilización coordinada, en la que confluyen

\footnotetext{
1 Este artículo ha sido escrito en el marco de la Red de Investigación R.A.M (Red Activismos Migrantes. Regímenes fronterizos y Derechos de Movilidad) financiada por el Plan Nacional de Investigación español (referencia RED2018-102783-T).
} 
diferentes colectivos de migrantes aglutinando diversas luchas en todo el territorio nacional. Esta movilización surge no sólo de las dificultades asociadas a la crisis sanitaria, sino de la toma de conciencia de su papel fundamental durante la pandemia, por ejemplo, en las tares de cuidado, en la producción y distribución de alimentos o en la provisión de servicios básicos. Como veremos, aunque las reclamaciones en torno a la regularización han formado parte de la agenda de las organizaciones de migrantes y de la sociedad civil durante varias décadas, esta reclamación coge fuerza y cristaliza en pleno confinamiento cuando el funcionamiento del país descansó, en gran medida, en el trabajo de las personas migrantes en estas tareas esenciales.

Su toma de conciencia como trabajadores esenciales, pero sin derechos, se ha visto alimentada adicionalmente por la respuesta del gobierno español ante la crisis sanitaria. A lo largo del mes de marzo y abril de 2020 el ejecutivo español articuló una serie de medidas excepcionales dirigidas a suavizar los efectos de la crisis económica ocasionada por la pandemia en los sectores más vulnerables de la población. Estas iniciativas, sin embargo, excluyeron a las personas migrantes en situación irregular impulsando y vertebrando una iniciativa de resistencia y de denuncia que ha cristalizado en una lucha política con elementos distintivos.

El objetivo de este artículo es analizar el surgimiento y desarrollo de esta movilización social, su composición, agenda y repertorio de actuación, así como sus efectos en la ciudadanía. El artículo se detendrá también en la respuesta del gobierno español durante la crisis sanitaria para entender el clima socio-político en el que surge. Este análisis, más que abordarse desde la literatura de los movimientos sociales ${ }^{2}$, se enmarca en los denominados estudios críticos de ciudadanía y más en concreto en la literatura sobre los actos de ciudadanía y la ciudadanía performativa de Engin Isin (2008, 2009, 2017, 2019), que a lo largo de la última década se ha enriquecido con los análisis sobre las luchas migrantes (Barbero, 2012; McNevin, 2012; López-Sala, 2019). Como veremos, las demandas de este colectivo se han articulado a través de una ciudadanía performativa orientada hacia el ejercicio de una voz política más allá del estatuto legal (que trasciende el estatuto legal), de la reclamación de derechos y de la articulación de formas de solidaridad con otros sujetos políticos. Han cristalizado así nuevas subjetividades políticas de estos sujetos móviles, nuevas formas de ser político que han vertebrado la forma y los contenidos de su reclamación de derechos en contra de las formas de injustica y desigualdad que han provocado las leyes de extranjería y las políticas fronterizas. Tras un brevísimo repaso de cómo han abordado los estudios de ciudadanía

Como ha subrayado recientemente Steinhilper, el activismo migrante ha sido escasamente abordado hasta la fecha por la literatura de los movimientos sociales en gran parte porque las teorías más influyentes en este campo -entre otras, la teoría de la movilización de recursos y el enfoque de la estructura de oportunidades políticas- consideran a los migrantes como actores "poco contenciosos" debido a los obstáculos legales, la escasez de recursos y las limitadas oportunidades políticas y discursivas (Steinhilper, 2018). 
las tensiones que emanan entre territorio y derechos como consecuencia de la creciente movilidad internacional, el artículo se detiene en el caso de las luchas ciudadanas del movimiento \#RegularizaciónYa desde esta perspectiva, unas luchas conformadas en el contexto de las especiales dificultades que ha ocasionado la crisis sanitaria en las personas migrantes, pero también en su toma de conciencia como sujetos esenciales que realizan una aportación imprescindible, pero que son objeto de no-derechos.

La información sobre la organización de este movimiento, su forma de trabajo y vinculación con otras organizaciones sociales se recopiló a través de los testimonios de sus portavoces en distintos medios de comunicación y en redes sociales. Estos instrumentos sirvieron también para identificar sus principales reivindicaciones, así como las acciones (actos) y hechos clave de la movilización. Para ello se analizaron los contenidos de la web de esta plataforma ${ }^{3}$, información que se contrastó con un análisis temático de su cuenta de twitter ${ }^{4}$. Además se emplearon artículos de prensa publicados por los periódicos El Diario, Público, El País y El Salto. La selección de estos periódicos se realizó no tanto por su línea editorial (criterio de diversidad) o su posicionamiento en el estudio general de medios $^{5}$ (criterio de impacto), sino debido a que han sido los medios escritos que han dado más cobertura informativa a esta campaña. El análisis ha cubierto todas las noticias (un total de 32) publicadas en torno a este movimiento social entre mediados de abril y principios de noviembre de 2020. Esta información ha permitido, adicionalmente, recoger el posicionamiento de este colectivo respecto a las actuaciones llevadas a cabo por el gobierno español a lo largo de la pandemia, así como desgranar los argumentos empleados en el ejercicio de su reclamación de derechos y mostrar los rasgos de la conformación de nuevas subjetividades políticas.

\section{Ciudadanía en tiempos de intensa movilidad internacional}

La ciudadanía ha sido generalmente concebida como un estatuto de pertenencia a una determinada comunidad política que conlleva la atribución de una serie de derechos y obligaciones. Tradicionalmente este estatuto se ha definido en torno a un determinado contorno jurisdiccional y territorial de la polity. A lo largo de las tres últimas décadas, las migraciones internacionales han retado esta noción de la ciudadanía, y la relación entre territorio y derechos ha sido un elemento crítico -y rupturista- en el campo de los estudios ciudadanos (Turner, 2016). Los estudios migratorios y la teoría política han centrado su interés

\footnotetext{
3 Véase <https://regularizacionya.com/>.

4 Para realizar este análisis temático se empleó la herramienta de búsqueda avanzada de esta red social que permite realizar múltiples búsquedas en base a una serie de parámetros (temas, hashtags, período temporal, etc.).

5 El País es el diario nacional de mayor tirada y El Diario y Público ocupan la tercera y la sexta posición entre los periódicos digitales nacionales. El Salto es un periódico digital que da amplia cobertura a la actualidad en materia de migraciones.
} 
en varias líneas de indagación: a) en las dimensiones globales y desterritorializadas de la ciudadanía, b) en el estatuto de los residentes no ciudadanos establecidos en los países de destino, así como en el estatuto de los ciudadanos no residentes; y, por último, c) en la ciudadanía externa y las relaciones Estado-diásporas. Aunque estas tres aproximaciones han permitido cerrar el círculo en torno a las dimensiones territoriales y los retos que en la ciudadanía ejerce la migración internacional, en todas ellas predomina una aproximación a la ciudadanía "como estatus", que subraya sus dimensiones normativas y la atribución de derechos por parte del Estado. La literatura en torno a los actos de ciudadanía, como una vía alternativa para investigar la ciudadanía, ha producido una ruptura analítica respecto a estos enfoques al considerar la ciudadanía no sólo como un estatus, sino, como una práctica, o incluso como una performance, expresada de varias formas, fundamentalmente en el lenguaje de una activa versus pasiva ciudadanía (Isin, 2009). Recientemente Isin ha argumentado que esta perspectiva performativa de la ciudadanía, como una reclamación de derechos, revela sus posibilidades transformativas (Isin, 2017) ${ }^{6}$. De acuerdo con esta perspectiva, como veremos a lo largo de los siguientes apartados, lo que hace de las luchas del movimiento \#RegularizaciónYa un ejemplo de ciudadanía performativa es su contestación sobre quién es sujeto de derechos, cómo en estas luchas incorporan a otros sujetos políticos, cómo se pone en acción esta ciudadanía a través de la reclamación de derechos (ciudadanía activa vs ciudadanía pasiva) y el ejercicio de "actos" y cómo estas luchas transforman las convenciones al aplicar principios de justicia e igualdad.

\section{Inmigrantes irregulares en tiempos de COVID. ¿Nadie va a quedarse atrás?}

\footnotetext{
"Nadie se va a quedar atrás en esta crisis sanitaria. Cuidaremos unos de otros a través de lo público. Combatiremos unidos para parar al virus y será así como venceremos" (Pedro Sánchez, Presidente del Gobierno de España. Comparecencia en el Congreso de los Diputados por el Estado de Alarma, 18 de marzo de 2020)

"Dicen que no van a dejar a nadie atrás, nosotros somos nadie, los negros, que estamos fuera de cualquier ayuda, porque ser irregular es como estar fuera de España, es como no formar parte de la sociedad porque no tenemos derechos" (Portavoz del Movimiento \#RegularizaciónYA, abril 2020) ${ }^{7}$
}

6 Este autor usa el concepto de ciudadanía performativa en cinco sentidos distintivos, pero solapados. En primer lugar la ciudadanía implica luchas sociales y políticas sobre quién puede y quién no puede actuar como sujeto de derechos; en segundo lugar esas luchas incluyen tanto a ciudadanos como no ciudadanos como actores relacionales; en tercer lugar la ciudadanía se pone en práctica a través de la reclamación de derechos; en cuarto lugar, los individuos ponen en práctica la ciudadanía a través del ejercicio de reclamaciones y del ejercicio de derechos y obligaciones; y en quinto lugar, a través de las luchas por sus derechos los individuos se constituyen como ciudadanos transformando las convenciones al aplicar principios como la igualdad, la justicia, la libertad, la emancipación y la solidaridad (Isin, 2017, p. 501-507).

7 Véase <https://www.elsaltodiario.com/migracion/lamine-saar-dicen-no-dejar-nadie-atras-peroparece-negros-somos-nadie-coronavirus $>$. 
La pandemia ha tenido efectos devastadores en las personas inmigrantes, particularmente entre aquellos que se emplean en la economía sumergida y los que se encuentran en situación irregular, que han visto seriamente afectados sus medios de subsistencia. Al igual que en otras geografías, las trabajadoras domésticas han sido uno de los colectivos que se han visto más afectadas por esta crisis ${ }^{8}$. El confinamiento estricto durante los primeros meses de la pandemia ${ }^{9}$, las dificultades económicas que han producido una reducción de las rentas de muchas familias y el temor al contagio ocasionaron la pérdida de empleo en algunos casos, así como, en otros, la reducción de las horas de trabajo y de la cuantía de los salarios ${ }^{10}$. La condición irregular de muchas de las mujeres inmigrantes del sector también obstaculizó el mantenimiento de sus empleos por la imposibilidad de justificar, por motivos laborales, sus desplazamientos enfrentándose a la posibilidad de recibir multas y a ser detenidas. A finales de marzo de 2020 el gobierno español aprobó un subsidio de desempleo excepcional dirigido a trabajadoras de este sector que hubieran perdido sus empleos como consecuencia de la crisis sanitaria y que les permitía recibir el $70 \%$ de sus ingresos habituales. Sin embargo, como denunciaron distintas organizaciones, esta iniciativa, cuyo anuncio causó grandes expectativas en las trabajadoras, redujo su cobertura a aquellas empleadas dadas de alta en este régimen de la seguridad social, excluyendo a las que trabajan informalmente y a las que se encuentran en situación irregular ${ }^{11}$. Esto ha supuesto que en la práctica esta medida sólo haya permitido cierto respaldo económico a un segmento reducido de las trabajadoras del sector. No debemos olvidar que el empleo doméstico y de cuidados es uno de los sectores donde la presencia de extranjeras es más abultada (Díaz-Gorfinkel, Martínez-Buján, 2018) y en el que, según distintas estimaciones, el trabajo informal alcanza aproximadamente un tercio del total (Zaguirre, 2019).

Otro de los colectivos que aglutina a inmigrantes irregulares seriamente afectados por esta crisis es el de los manteros. Los manteros son un colectivo integrado por inmigrantes africanos que se dedican al comercio informal

${ }_{8}$ Véase <https://www.hrw.org/news/2020/05/01/it-time-world-start-caring-caregivers>.

9 Entre mediados de marzo y finales de abril de 2020 el gobierno español estableció un confinamiento domiciliario estricto de los ciudadanos que incluyó algunas excepciones por causa de fuerza mayor como salidas para adquirir alimentos, desplazarse a un centro sanitario o por razones laborales. Entre finales de marzo y mediados de abril de 2020 sólo pudieron abandonar este confinamiento domiciliario los considerados trabajadores esenciales.

${ }^{10}$ En el caso de las trabajadoras internas, empleadas mayoritariamente en el cuidado de personas de avanzada edad, muchas han sufrieron la pérdida del empleo y de la vivienda por el fallecimiento por Covid de los ancianos que cuidaban. Además, muchas empleadas quedaron encerradas en sus puestos de trabajo, con jornadas mucho más extensas, sin horas libres y sin pago de horas extras. Las dificultades específicas a las que se enfrentaron este tipo de trabajadoras impulsaron algunas iniciativas de apoyo mutuo y solidaridad durante los primeros meses de la pandemia, que incluyeron, entre otras, la creación por parte de algunas organizaciones de cajas de resistencia o iniciativas de asesoramiento jurídico dirigidas a las trabajadoras del sector. Algunas de estas iniciativas pueden verse en: < https://www.elsaltodiario.com/coronavirus/empleadas-hogar-tra bajo-domestico-esencial-organizan-ante-pandemia $>$.

${ }^{11}$ Véase <https://elpais.com/economia/2020-08-23/el-subsidio-que-no-llega-ni-a-las-mas-afortunadas.html> . 
ambulante en el centro de grandes ciudades, como Madrid y Barcelona, y en los municipios turísticos ${ }^{12}$. El confinamiento, y por lo tanto la imposibilidad de salir y de desplazarse, así como el desplome del turismo, tanto en las grandes ciudades como en las zonas de litoral, ha impedido o limitado de forma muy aguda la continuidad de su actividad laboral. Se trata de un colectivo muy precarizado, cuyos ingresos dependen de la venta diaria, que sin embargo está ampliamente cohesionado y organizado a través del denominado Sindicato de Manteros ${ }^{13}$, que cuenta con una importante trayectoria de lucha política dirigida a conseguir derechos laborales y ciudadanos ${ }^{14}$.

A pesar del aumento de la demanda de trabajadores para el sector agrícola durante las campañas de 2020, debido a las limitaciones de la llegada de trabajadores desde el exterior, tanto a través de programas bilaterales como de la libertad de circulación en el espacio europeo, los migrantes sinpapeles no han podido transformar esta situación en la posibilidad de trabajar formalmente en la agricultura y regularizar su situación. En abril de 2020 el gobierno español, ante las presiones de los empresarios del sector, aprobó una serie de medidas extraordinarias dirigidas a favorecer la contratación temporal de trabajadores para la agricultura. El decreto donde se concretaban dichas medidas incluía, por ejemplo, un mecanismo de flexibilización temporal que permitía compatibilizar el trabajo agrario con la prestación por desempleo, la contratación de jóvenes de entre 18 y 21 años nacionales de terceros países que estuvieran en situación regular, así como la de inmigrantes cuyos permisos de trabajo concluyeran entre la declaración del estado de alarma a mediados del mes de marzo y el 30 de junio de 2020. El decreto, sin embargo, no recogía la posibilidad de contratar personas inmigrantes en situación irregular, que en muchos casos llevaban años trabajando informalmente en distinto enclaves de este sector. De esta forma se ha dado la paradoja de que el aumento de la demanda en el sector y las iniciativas estatales dirigidas a impulsar el empleo en el sector agrícola se han compaginado con la presencia de un alto número de trabajadores en situación irregular que

\footnotetext{
${ }^{12}$ La denominación de manteros procede de la venta ambulante de productos empleando una manta que colocan en el suelo. Otras formas de denominar esta actividad es la de TopManta, un término que surge porque muchos de estos trabajadores ambulantes comenzaron a realizar su actividad a principios de la década pasada vendiendo CDs y DVD musicales, para posteriormente especializarse en la venta de productos textiles. Las reivindicaciones de este colectivo se han basado en la defensa de su trabajo como una actividad productiva, en la regularización y en el ejercicio del derecho a la ciudad (véase Molinero, Avallone, 2020).

${ }^{13}$ Este sindicato se crea en Cataluña en 2015 después de una persecución policial en el que falleció uno de los vendedores. El sindicato se crea para ofrecer asesoramiento laboral, sanitario y legal a sus integrantes. También se ha convertido en un instrumento muy efectivo para denunciar la criminalización y la persecución policial a la que se les somete.

${ }^{14}$ Durante el confinamiento el Sindicato de Manteros hizo frente a la falta de ingresos que supuso la imposibilidad de mantener su actividad comercial a través de un crowdfunding popular que les permitió crear un banco de alimentos -dirigido no sólo a miembros del sindicato, sino a otros vecinos con necesidades- y una caja de resistencia.
} 
tras años empleándose en el sector, sin embargo, no han podido acceder a un trabajo formal en la agricultura, y por tanto regularizarse, debido a las limitaciones impuestas por el gobierno.

Los problemas vinculados a la crisis sanitaria y las limitaciones de movilidad también han afectado a los solicitantes de asilo que se encuentran en España. Por un lado, el cierre de la administración durante el estado de alarma produjo una paralización de la gestión y por tanto la demora en el acceso al procedimiento y en la tramitación de los expedientes en curso. A ello se une la incertidumbre de aquellos que se encontraban en los recursos de acogida, tanto municipales como estatales, que estaban al límite de su capacidad y las dificultades para encontrar trabajo debido a la grave crisis de empleo derivada de la pandemia (Comisión Española de Ayuda al Refugiado, 2020). Esta situación se ha agravado aún más entre los solicitantes que se encuentran en las ciudades de Ceuta y Melilla. La política española ha limitado la derivación a la península de los solicitantes de asilo mientras se tramita su estatuto, salvo casos muy excepcionales de perfiles extremadamente vulnerables (López-Sala, 2020). Esto ha supuesto en la práctica su inmovilización en estos enclaves y su acogida en los Centros de Estancia Temporal de Migrantes $(\mathrm{CETIS})^{15}$. Aunque estos centros son instalaciones en régimen abierto se encuentran ampliamente sobreocupadas. Los problemas de hacinamiento en los CETIs durante la pandemia y los peligros que ello puede ocasionar para la salud de sus residentes han sido objeto de denuncias constantes de las organizaciones sociales (véase Comisión Española de Ayuda al Refugiado, 2020; Arce, 2020).

Las organizaciones que aglutinan a estos colectivos (asociaciones de empleadas domésticas, sindicato de manteros, organizaciones de temporeros agrícolas, de solicitantes de asilo, etc.) han denunciado que el efecto de la pandemia ha sido especialmente intenso entre la población de origen extranjero y que este efecto se ha amplificado aún más en el caso de los que carecen de un estatuto legal regular. No hay que olvidar que durante los primeros meses de la pandemia el ejecutivo español adoptó, bajo el lema "nadie va a quedarse atrás", una serie de medidas de carácter económico y social dirigidas a paliar los efectos de la pandemia en la población. Estas medidas incluyeron el establecimiento del Ingreso Mínimo Vital (IMV), un seguro de renta mínima para las personas y familias de menores ingresos, la supresión de los desahucios, ayudas a los trabajadores autónomos y a los trabajadores sometidos a expedientes de regulación de empleo temporal, la ampliación de los subsidios de desempleo, así como el seguro de desempleo destinado a trabajadoras domésticas previamente mencionado. Ninguna de estas medidas incluían entre sus destinatarios a trabajadores que se emplean en la economía sumergida y a personas inmigrantes en situación irregular.

\footnotetext{
${ }^{15}$ Los CETIs (Centros de Estancia Temporal de Inmigrantes) son centros de acogida localizados en las ciudades de Ceuta y Melilla que dependen del Ministerio de Trabajo.
} 


\title{
\#RegularizaciónYa
}

\author{
"Si somos actividad esencial, nuestros derechos también lo son"16 \\ "Esta vez seremos las personas migrantes quienes tomamos las riendas \\ en la lucha por nuestros derechos" (Declaraciones de la campaña \\ \#RegularizacionYa) ${ }^{17}$
}

La asunción de que el trabajo realizado por las personas inmigrantes irregulares durante la crisis sanitaria estaba siendo determinante para mantener el funcionamiento del país y su visión de que las medidas adoptadas por el gobierno español "iban a dejarles atrás" se convirtieron en elementos catalizadores del inicio de la acción política y la lucha por los derechos de un amplio abanico de organizaciones de migrantes y refugiados más o menos formalizadas. Las regularizaciones anunciadas por Portugal e Italia también se convirtieron, en un primer momento, en un elemento de referencia para articular esta demanda ${ }^{18}$.

Aunque en la historia inmigratoria reciente de España hay varias experiencias de luchas políticas de los migrantes sin papeles que finalmente terminaron en un proceso de regularización, como la que tuvo lugar en 2001 (Barbero, 2012), esta movilización presenta algunos elementos distintivos respecto a las anteriores y muestra, como estas luchas sociales y políticas se expresan en el lenguaje de la ciudadanía performativa. Se trata de un movimiento que aglutina por primera vez inmigrantes irregulares de distintas organizaciones formales e informales en todo el territorio nacional y pertenecientes a distintos tipos de colectivos y sectores. Es, por tanto, un movimiento autoorganizado de amplio alcance en términos geográficos y sectoriales que se inicia en abril de 2020 con el lanzamiento de la campaña \#RegularizaciónYa. Este acto inaugural empleó principalmente las redes sociales, en pleno estado de alarma y de confinamiento estricto, como vehículo para demandar, a través de una carta redactada por cien organizaciones de inmigrantes y dirigida al gobierno español y al defensor del pueblo, una regularización de todos los inmigrantes y refugiados en España. Se trata, por tanto, de una acción que subraya el ejercicio de la ciudadanía como práctica y que se pone en acción a través de la reclamación de derechos.

Esta primera iniciativa se acompañó de una amplia campaña de difusión vehiculizada a través de redes sociales y dirigida a buscar apoyos entre las organizaciones de la sociedad civil con el objetivo de incorporar en sus luchas

\footnotetext{
${ }_{16}$ Véase <https://www.huffingtonpost.es/entry/las-empleadas-del-hogar-responden-al-gobierno-sisomos-actividad-esencial-nuestros-derechos-tambien-lo-son_es_5e84afedc5b6871702a83746>.

17 Véase $<$ https://regularizacionya.com/>.

${ }^{18}$ En el caso de Portugal esta regularización se dirigió, en exclusiva, a las personas que tuvieran en curso una solicitud de permiso de trabajo y residencia. La intención del gobierno luso fue favorecer el acceso de las personas en situación irregular al sistema sanitario y a subsidios de desempleo. En el caso italiano la regularización se dirigió a trabajadores temporeros y de cuidados e incluía un permiso de trabajo de seis meses con posibilidad de prórroga (PICUM, 2020).
} 
a otros sujetos políticos. En sólo una semana, la demanda de esta alianza de colectivos migrantes recibió un gran respaldo a través de la adhesión de más de mil organizaciones, entre las que se encuentran las más importantes en la defensa de los derechos humanos y sociales. Se trata, por tanto, de un movimiento autónomo, donde han confluido las luchas de distintos sectores de la inmigración; y que a pesar de surgir de personas inmigrantes sin papeles ha recibido, en el contexto social, político y económico de la crisis sanitaria, un abultadísimo apoyo por parte de la sociedad civil. Sin embargo, este movimiento muestra también elementos similares a los observados en las luchas previas de los sinpapeles en España. En particular, el aunar en sus reivindicaciones el acceso a un estatuto legal y la defensa de sus derechos laborales; pero también la reivindicación de una ciudadanía -y de una forma de ejercer la ciudadanía- que no pase, en exclusiva, por la atribución de derechos por parte del Estado, sino que emane de la "presencia-residencia" y de la participación en la sociedad española.

Además de demandar una regulación sin condiciones, urgente y permanente la reivindicación de este movimiento social ha incorporado otros elementos que conforman su agenda política entre los que se incluyen, la resolución favorable y la renovación de las autorizaciones de residencia y trabajo, de las solicitudes de reagrupación familiar y de las solicitudes de asilo y protección internacional en trámite. También han demandado el cierre definitivo de los centros de internamiento y el archivo de los expedientes de devolución y expulsión, el traslado urgente a la península de las personas que se encuentran en los centros de estancia temporal de Ceuta y Melilla y el fin de las devoluciones en caliente y de las detenciones por perfil étnico.

Por último, este movimiento social considera que las circunstancias excepciones que han emanado de la crisis sanitaria convierten el momento actual en una oportunidad histórica para dar respuesta política a la situación de miles de personas inmigrantes irregulares que se emplean en la economía sumergida, cuyo trabajo se ha revelado como esencial para el funcionamiento del país, un aspecto reconocido por la propia clase política. Concluyen, además, que la propia ley de extranjería les ampara en esta demanda ${ }^{19}$, por criterios de interés público.

El siguiente paso de este movimiento social, tras recibir un amplio apoyo de la sociedad civil, fue dirigir sus esfuerzos a incluir esta demanda en el debate político a través de diversas acciones institucionales. En primer lugar esta reclamación de derechos fue compartida por miembros de importantes organizaciones de la sociedad civil (CEAR, Cáritas, Oxfam) en la Comisión de Reconstrucción Económica y Social del Congreso, una comisión creada en el Parlamento español para abordar y discutir medidas dirigidas a mitigar los efectos de la crisis

\footnotetext{
${ }^{19}$ El artículo 127 del reglamento de la Ley de Extranjería española indica que se pueden conceder autorizaciones de residencial temporal por circunstancias excepcionales de colaboración con las autoridades, razones de seguridad nacional o interés público.
} 
ocasionada por la Covid-19. Pero la acción más importante fue la elaboración por parte de este movimiento y la posterior presentación, tras recibir el apoyo de varios partidos políticos del arco parlamentario ${ }^{20}$, de una Proposición No de Ley de Regularización ${ }^{21}$ en el Congreso español en junio de 2020. El registro en el congreso de esta iniciativa se acompañó de diversas concentraciones a lo largo de todo el territorio nacional a finales de junio de 2020, que se prolongaron durante los meses de verano, cuando el fin del confinamiento permitió el acceso a espacios públicos y la visibilización de sus demandas a través de repertorios de acción más convencionales.

Frente a estas reivindicaciones el gobierno español se ha mostrado reiteradamente contrario a adoptar una medida de esta naturaleza ${ }^{22}$. Tras no pronunciarse durante el recorrido de los primeros meses de esta campaña, o posteriormente, alegar que esta regularización era inviable debido a los acuerdos migratorios adoptados en Europa, la entrada de esta cuestión en el debate público y político en junio de 2020 se acompañó de declaraciones del Ministro de Inclusión Seguridad Social e Inmigración y de la Secretaria de Estado de Inmigración indicando que esta iniciativa no formaba parte de su agenda. La posición que ha defendido el partido mayoritario del gobierno, el Partido Socialista Obrero Español, es que la política migratoria española debía incentivar una migración regular, segura y ordenada. En el mes de junio de 2020 se adoptaron además una serie de decisiones que en opinión del ejecutivo podían tener efectos más positivos para los migrantes que realizar una regularización extraordinaria ${ }^{23}$. Esta postura ha contrastado con la de Unidas Podemos, su socio de gobierno, uno de los partidos que ha defendido dicha regularización y que apoyó en el Parlamento la Proposición no de Ley, por lo que la gestión de la inmigración irregular en tiempos de pandemia ha producido amplias tensiones en el seno del gobierno.

${ }^{20}$ Los partidos que apoyaron y registraron esta Proposición No de Ley, fueron Unidas-Podemos (que forma parte del gobierno de coalición en estos momentos), Más País, Compromís, Esquerra Republicana de Catalunya, Junts per Catalunya, EH Bildu, Bloque Nacionalista Gallego y la CUP.

${ }^{21}$ Esta Proposición no de Ley insta al gobierno, como se mencionó previamente, a aplicar el artículo 127 del Reglamento de Extranjería que permite autorizar la residencia por razones de interés público y seguridad nacional y la concesión de permisos de una duración de cinco años para todos los inmigrantes irregulares que se encuentren en territorio nacional que permita la actividad laboral por cuenta propia o ajena sin ningún tipo de limitación por sectores o territorios. Según esta Proposición no de Ley, los únicos requisitos que se demandarían a los migrantes que pudieran acogerse sería la acreditación de la identidad y el empadronamiento, así como la acreditación a través de cualquier documento de su estancia en España antes del 14 de marzo de 2020.

${ }^{22}$ Durante sus dos primeras décadas como país de inmigración España llevó a cabo varios procesos de regularización extraordinaria (1986, 1991, 1996, 2000, 2001). La última regularización se produjo en 2005.

${ }^{23}$ Entre estas medidas se encontraban la rebaja de los requisitos económicos para los procesos de reagrupación familiar y para las renovaciones de permisos de las solicitudes realizadas antes del estado de alarma, así como la concesión de autorizaciones de trabajo a los menores extranjeros no acompañados. Además en septiembre de 2020 se aprobó una instrucción para flexibilizar los requisitos exigidos para obtener un permiso de residencia de cinco años a los inmigrantes extracomunitarios con hijos españoles menores de edad. 
A pesar del agitado debate que acompañó esta propuesta parlamentaria la Proposición No de Ley fue finalmente rechazada en el Congreso en septiembre de 2020, el mismo día en el que se anunció la reapertura de los Centros de Internamiento de Extranjeros cerrados desde abril de 2020 como consecuencia de la crisis sanitaria. Esto ha producido cierta decepción en el seno de este movimiento, que considera que se ha perdido una oportunidad histórica para reconocer sus derechos y que el gobierno ha dado la espalda a miles de inmigrantes que viven en España en el contexto de la enorme crisis social, económica y de salud que ha ocasionado esta pandemia ${ }^{24}$. Sin embargo consideran un éxito que la iniciativa contara con un apoyo mayoritario de la sociedad civil y que llegara al Parlamento. Por otro lado, la respuesta del gobierno ante estas reclamaciones ha producido que parte de la sociedad civil percibiera este rechazo a la regularización como un indicio más de que la intención del actual gobierno es mantener una política continuista con la desarrollada por el anterior ejecutivo conservador. En los últimos meses este movimiento ha mantenido sus reivindicaciones y su movilización a través del apoyo de distintos diputados en la Comisión de Trabajo, Seguridad Social, Migraciones e Inclusión del Parlamento, ha realizado reuniones con diversos representantes de organismos internacionales, como el Alto Representante para los Derechos de los Migrantes de las Naciones Unidas, e iniciado otras acciones más clásicas dentro de los repertorios de los movimientos sociales como concentraciones y manifestaciones en alianza con organizaciones de defensa de los derechos de los migrantes. En su agenda se mantiene su objetivo fundamental, pero su iniciativa se está articulando también a través de objetivos más específicos, particularmente importantes en el contexto actual, como el acceso de las personas inmigrantes en situación administrativa irregular al Ingreso Mínimo Vital y la eliminación del requisito de empadronamiento para articular este acceso.

\section{Conclusiones}

La Covid-19 ha provocado una profunda crisis social, económica y política en España que ha infligido una importante herida en las condiciones de vida de la población, pero especialmente en los sectores más vulnerables, entre los que destacan las comunidades inmigrantes. Durante los meses de confinamiento y a lo largo del tiempo en que se han aplicado restricciones en la movilidad ha quedado patente el papel fundamental que cumplen los migrantes en algunos sectores considerados esenciales como la producción de alimentos, las tareas de cuidado, los servicios de limpieza y mantenimiento o las cadenas de distribución de mercancías. Su condición de trabajadores esenciales contrasta, paradójicamente, con algunos elementos estructurales en estos sectores económicos, especialmente los altos niveles de informalidad y la presencia de un destacado número de trabajadores migrantes en situación administrativa irregular. Ha sido precisamente su toma de

${ }^{24}$ Véase $<$ https://regularizacionya.com/ $>$. 
conciencia como sujetos esenciales que realizan una aportación imprescindible, pero que son objeto de no-derechos, el elemento catalizador de la aparición de una amplia movilización de las personas inmigrantes sinpapeles en España conformando una iniciativa de resistencia y de denuncia que ha cristalizado en una lucha política con elementos distintivos en torno al movimiento \#RegularizaciónYa.

El movimiento \#RegularizaciónYa ha puesto en escena una ciudadanía performativa que interroga su relación y su estatuto en el seno del régimen ciudadano español y que, como propone esta aproximación analítica, refleja formas de escenificación de esta ciudadanía más allá de estatutos atribuidos y derechos formales a través de la práctica de una ciudadanía activa (Isin, 2009, 2017), mostrando, por añadidura, cómo la ciudadanía está sujeta a una reinvención permanente (Balibar, 2004).

En el seno de esta "ciudadanía performativa" (Isin, 2017, 2019), según la cuál, en sus propias palabras "la ciudadanía es cualquier cosa menos algo estable" y "como la gente pone en práctica su ciudadanía juega un papel fundamental en contestar y construir ciudadanía y vincular significado a los derechos" (Isin, 2017, p. 501-502), el artículo se ha detenido en las luchas y reclamaciones de derechos de las personas inmigrantes en situación irregular en el contexto de la pandemia. El análisis del movimiento \#RegularizaciónYa puede ser considerado un ejemplo paradigmático de ciudadanía performativa en: a) su contestación sobre quien es sujeto de derechos - los migrantes sinpapeles, sin estatuto y sin derechos ciudadanos formalmente atribuidos; b) cómo en estas luchas incorporan a otros sujetos políticos - la sociedad civil y ciertos sectores de la clase política como ha ejemplarizado el respaldo de la campaña impulsada por este movimiento y el apoyo que recibió la Preposición no de Ley; c) cómo pone en acción esta ciudadanía a través de la reclamación de derechos y actos - rastreada a través del análisis de sus reclamaciones, iniciativas y repertorio de acción incluso en tiempos de pandemia y de confinamiento estricto; y d) cómo estas luchas transforman las convenciones al aplicar principios de justicia e igualdad.

\section{Referencias bibliográficas}

ANDERSON, Bridget; POESCHEL, Friedrich; RUHS, Martin. COVID-19 and systemic resilience: What role for migrant workers? Ginebra: Organización Internacional de las Migraciones, 2020.

ARCE, Carlos. Migrants in Spain in the time of COVID-19: challenges and opportunities. Florencia: Migration Policy Centre. European University Institute, 2020.

BALIBAR, Étienne. We, the people of Europe? Reflections on transnational citizenship. Princeton: Princeton University Press, 2004.

BARBERO, Iker. Expanding Acts of Citizenship: The Struggles of Sinpapeles Migrants. Social \& Legal Studies, v. 21, n. 4, p. 529-547, 2012.

COMISIÓN ESPAÑOLA DE AYUDA AL REFUGIADO (CEAR). Situación de las personas en necesidad de protección internacional ante la COVID 19. Madrid: CEAR, 2020. 
DíAZ-GORFINKEL, Magdalena; MARTíNEZ-BUJÁN, Raquel. Mujeres migrantes y trabajos de cuidados: transformaciones del sector doméstico en España. Panorama Social, v. 27, p. 105-118, 2018.

ISIN, Engin. Theorizing acts of citizenship. In: ISIN, Engin; NIELSEN, Greg (eds.). Acts of Citizenship. Londres: Palgrave Macmillan, 2008, p. 15-43.

ISIN, Engin. Citizenship in Flux: the Figure of the Activist Citizen. Subjectivity, v. 29, p. 367-388, 2009.

ISIN, Engin. Performative Citizenship. In: SHACHAR, Ayelet et al. (eds.). The Oxford Handbook of Citizenship. Oxford: Oxford University Press, 2017, p. 500-523.

ISIN, Engin. Doing Rights with Things. The Art of becoming Citizens. In: HILDEBRANDT, Paula et al. (eds.). Performing Citizenship: Bodies, Agencies, Limitations. Londres: Palgrave, 2019, p. 45-56.

LASTRA, María; CACHÓN, Lorenzo. Determinantes de la movilidad ocupacional segmentada de los inmigrantes no comunitarios en España. Revista Internacional de Sociología, v. 71, n. 2, p. 383-413, 2013.

LÓPEZ-SALA, Ana. "You're not getting rid of us". Performing acts of citizenship in times of emigration. Citizenship Studies, v. 23, n. 2, p. 97-114, 2019.

LÓPEZ-SALA, Ana. Keeping up appearances: dubious legality, fundamental rights and migration control at the peripheral borders of Europe. The cases of Ceuta and Melilla. In: CARRERA, Sergio; STEFAN, Marco (eds.). Fundamental Rights Challenges in Border Control and Expulsion of Irregular Immigrants in the European Union. Complaint mechanisms and Access to Justice. London: Routledge, 2020, p. 25-42.

McNEVIN, Anne. Undocumented citizens. Shifting grounds of citizenship in Los Angeles. In: NYERS, Peter; RYGIEL, Kim (eds.). Citizenship, Migrant Activism and the Politics of Movement. London: Routledge, 2012, p. 165-183.

MOLINERO, Yoan; AVALLONE, Gennaro. El trabajo ambulante: entre derecho a la ciudad y represión: El caso de la resistencia de los trabajadores senegaleses en la ciudad de Salerno. Migraciones, n. 48, p. 21-50, 2010.

NEWLAND, Kathleen. Will International Migration Governance Survive the COVID-19 Pandemic?. Washington: Migration Policy Institute, 2020.

PAPADEMETRIOUS, Demetrios; HOOPER, Kate. How is COVID-19 Reshaping Labour Migration? International Migration, v. 58, n. 4, p. 259-263, 2020.

PICUM. Non-exhaustive overview of European government measures impacting undocumented migrants taken in the context of COVID-19. Bruselas, 2020.

STEINHILPER, Elias. Mobilizing in transnational contentious spaces: linking relations, emotions and space in migrant activism. Social Movement Studies, v. 17, n. 5, p. 574-591, 2018.

TURNER, Bryan. We are all denizens now. On the erosion of citizenship. Citizenship Studies, v. 20, n. 6-7, p. 679-692, 2016.

ZAGUIRRE, Arantxa. Empleadas del hogar: un caso evidente de discriminación indirecta. Zoom Social, n. 3, p. 1-16, 2019. 